Supporting Information

\title{
Few-Layer Tin Sulfide: A New Black-Phosphorus-Analogure 2D Material with Sizeable Band Gap, Odd-Even Quantum Confinement Effect, and High Carrier Mobility
}

Chao Xin, ${ }^{\#, \dot{\dagger}}$ Jiaxin Zheng, ${ }^{\#, \dot{ }}$ Yantao Su, ${ }^{\dagger}$ Shuankui Li, ${ }^{\dagger}$ Bingkai Zhang, ${ }^{\dagger}$ Yancong Feng $^{\dagger}$ and Feng Pan ${ }^{\dagger}{ }^{\dagger}$

${ }^{\dagger}$ School of Advanced Materials, Peking University Shenzhen Graduate School, Shenzhen 518055, China

\section{Corresponding Author}

*E-mail: panfeng@pkusz.edu.cn (F. Pan). Tel: +86-755-26033200

\#These authors contributed equally. 


\section{Supporting Information Figures}

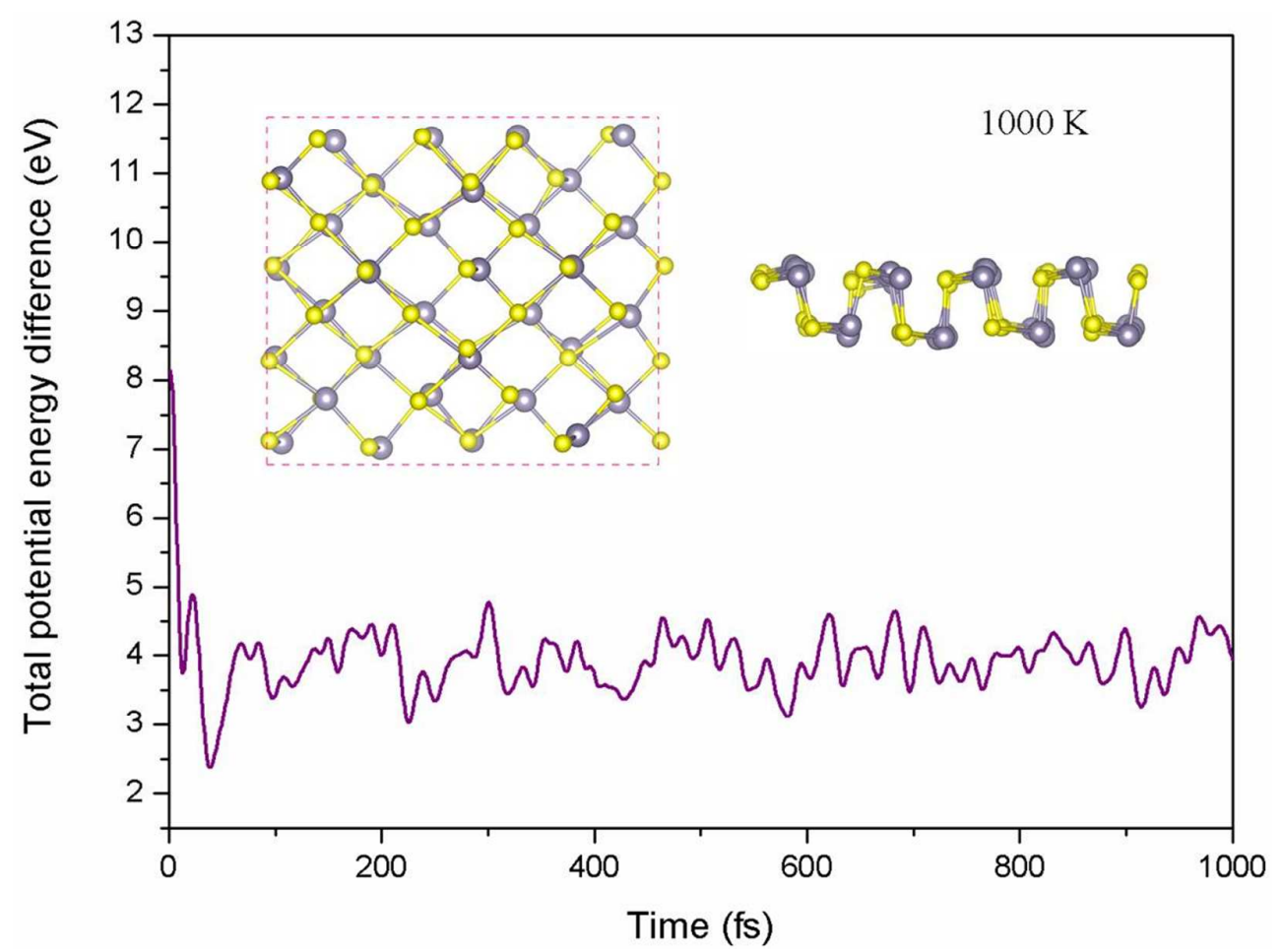

Figure SI1. Tolal potential energy fluctuation during AIMD simulations of monolayer $\mathrm{SnS}$ at $1000 \mathrm{~K}$. The insets show the snapshot at $1 p s$ for $4 \times 4$ supercell simulation. 

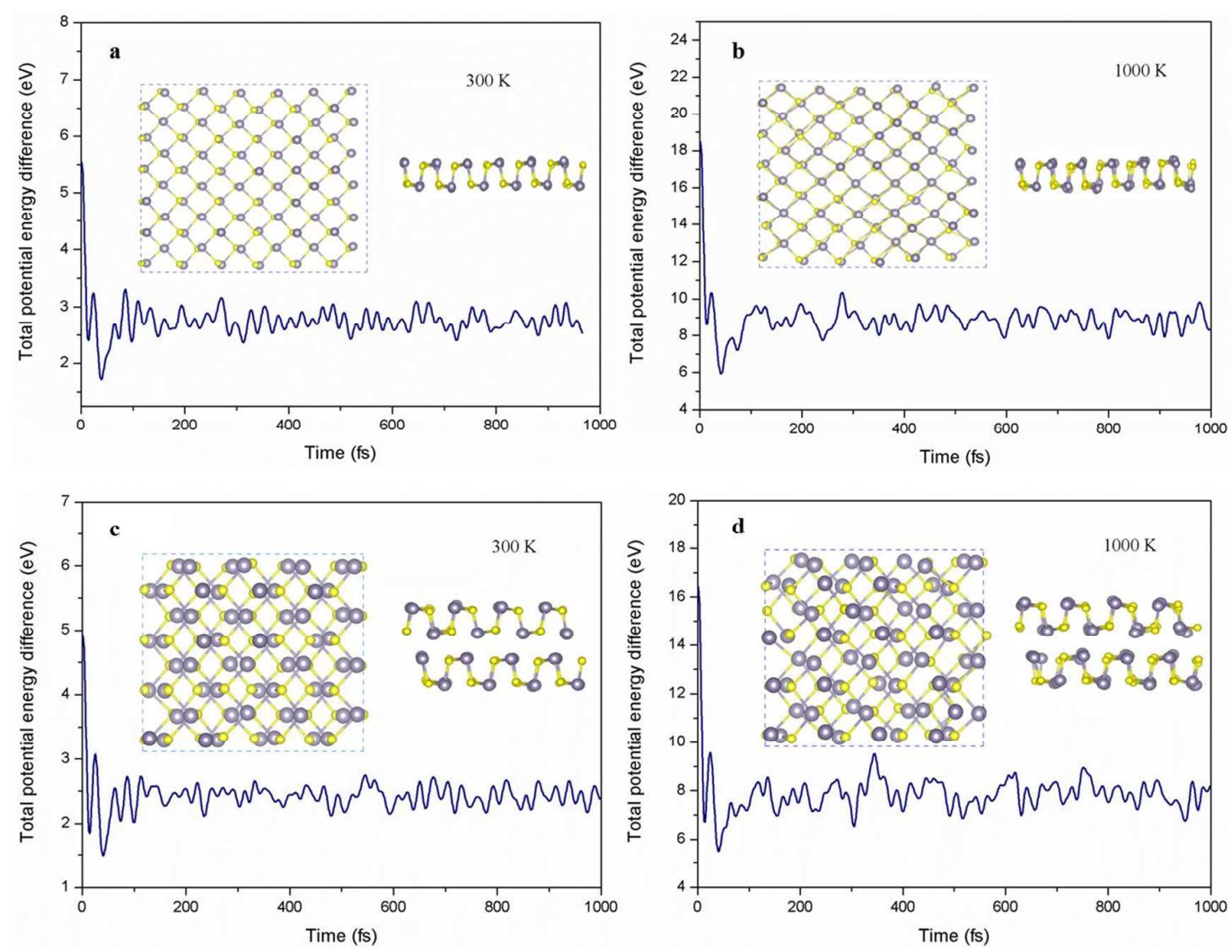

Figure SI2. Total potential energy fluctuation during AIMD simulations of SnS. (a,b) Monolayer at $300 \mathrm{~K}$ and $1000 \mathrm{~K}$. (c,d) Bilayer at $300 \mathrm{~K}$ and $1000 \mathrm{~K}$. The insets show the snapshot at $1 p s$ for monolayer $6 \times 6$ and bilayer $4 \times 4$ supercell simulation. 

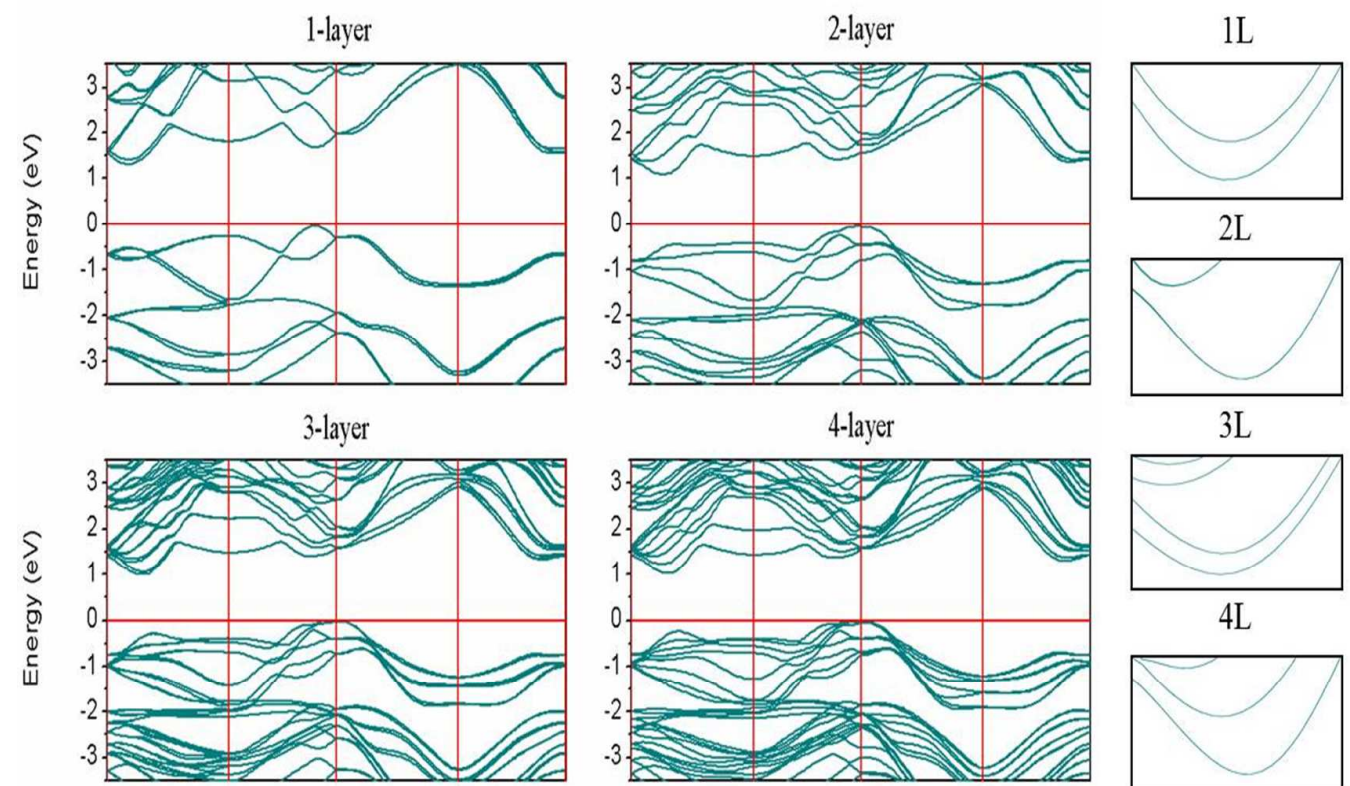

3L
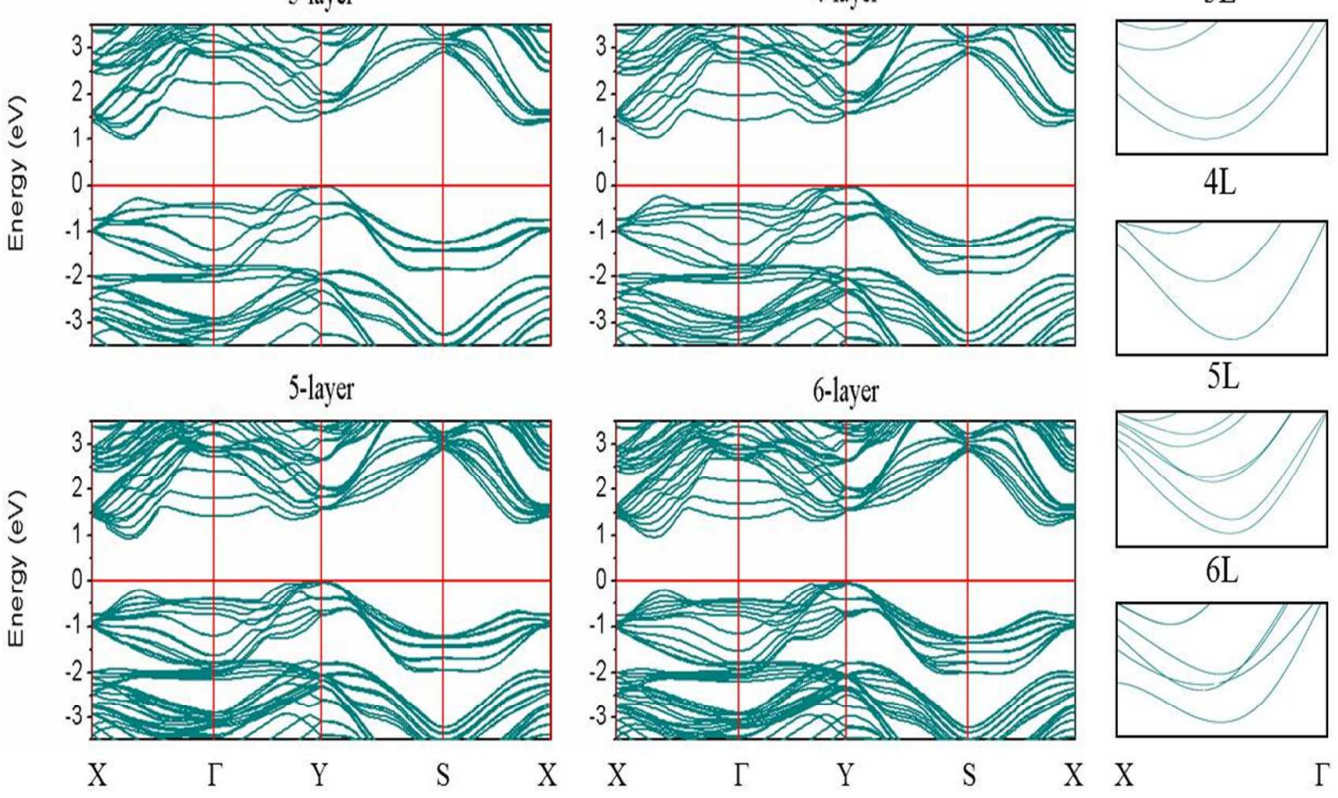

$4 \mathrm{~L}$

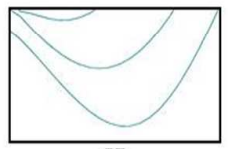

$5 \mathrm{~L}$
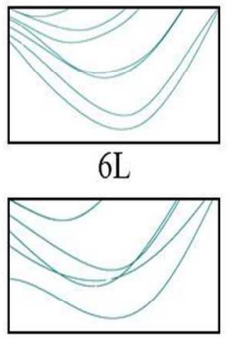

$\mathrm{X}$

Figure SI3. Electronic band structures for bulk and few-layer (1-6) SnS. The magnified plotting of conduction-band minimum was placed at the right. Results were calculated using the PBE with SOC. 

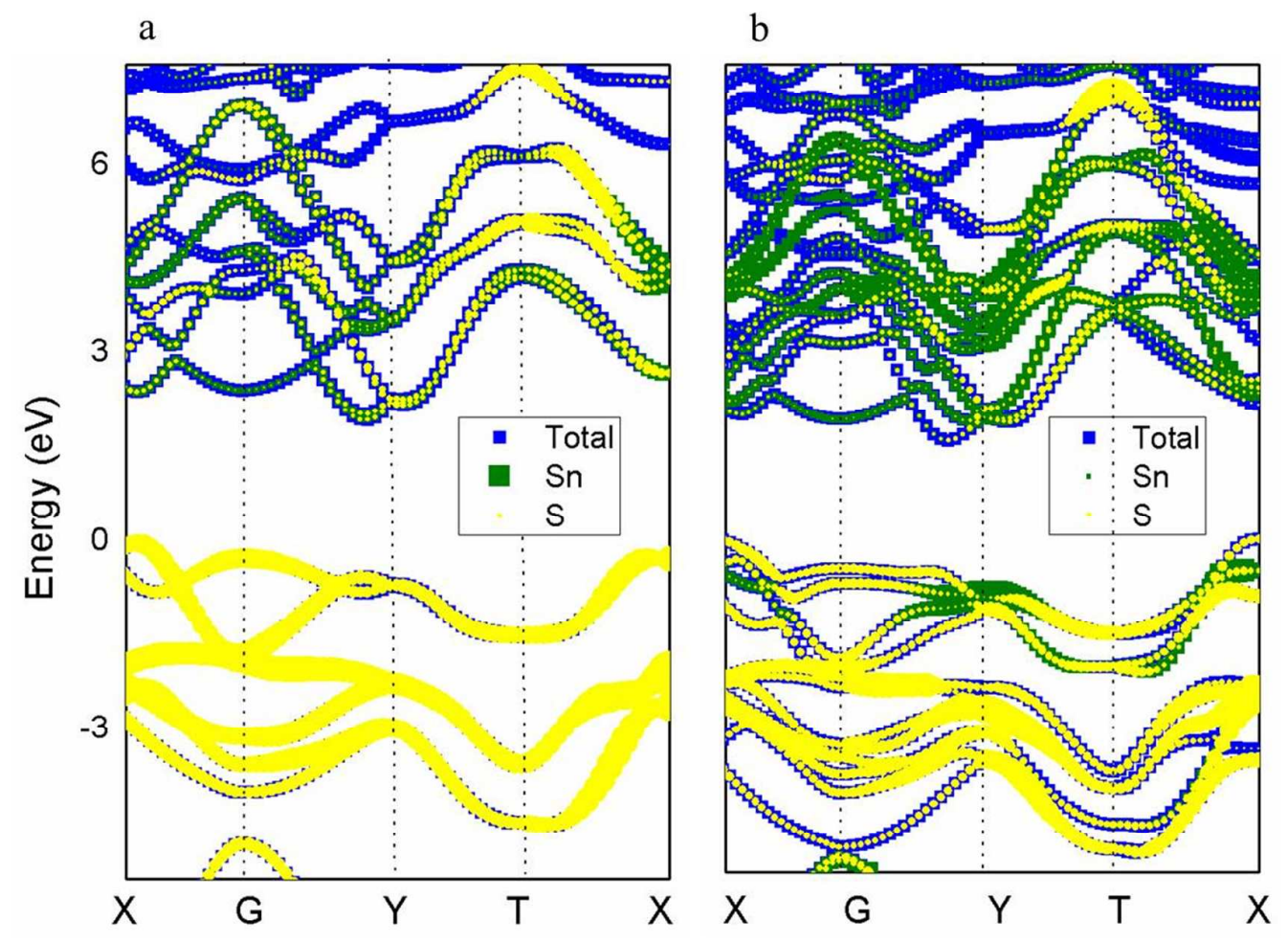

Figure SI4. Projection band structure of SnS. (a) monolayer, (b) bilayer. 

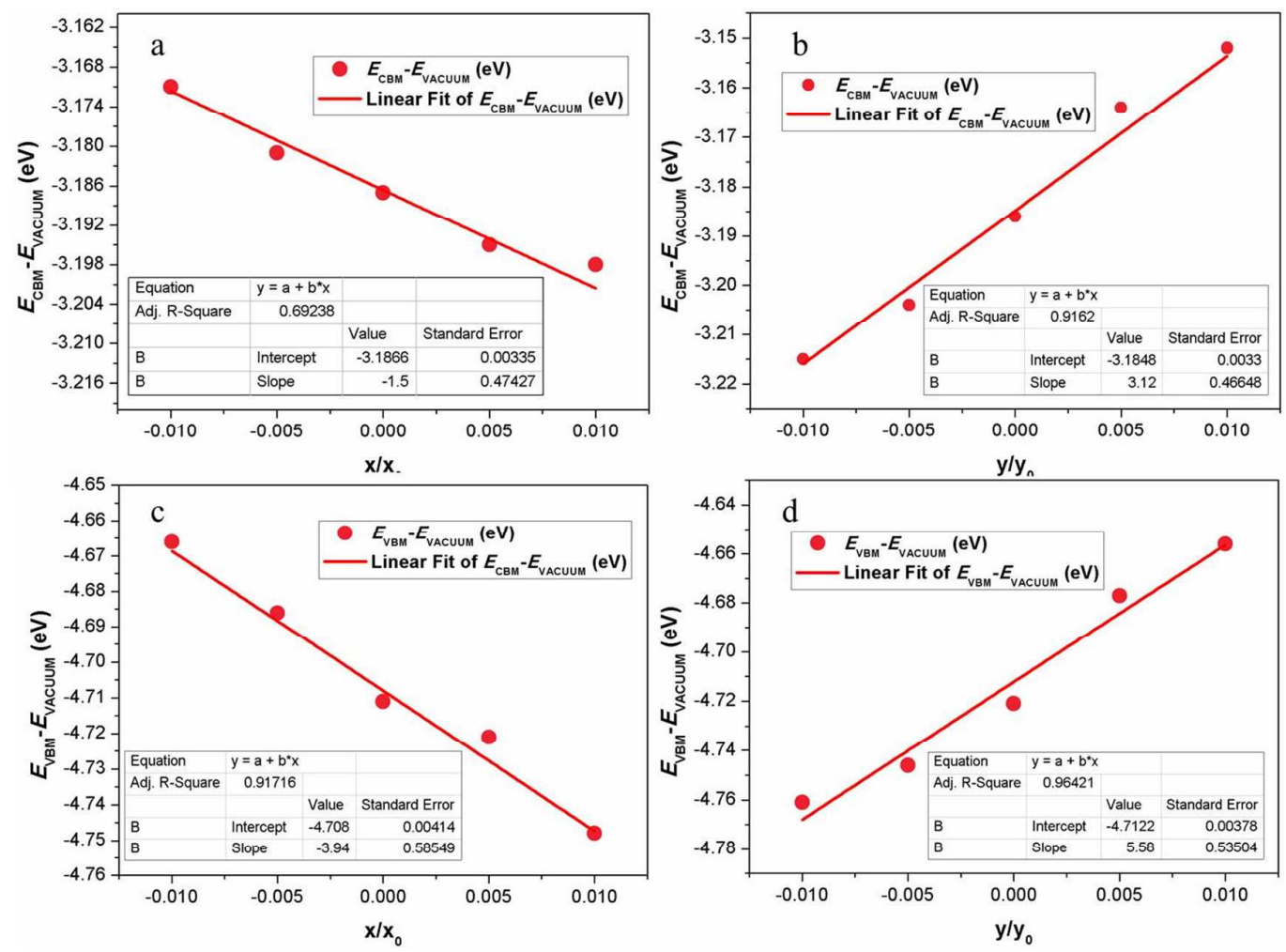

Figure SI5. Band energy of monolayer SnS. (a,b) The CBM of monolayer SnS. (c,d) The VBM of monolayer SnS. 

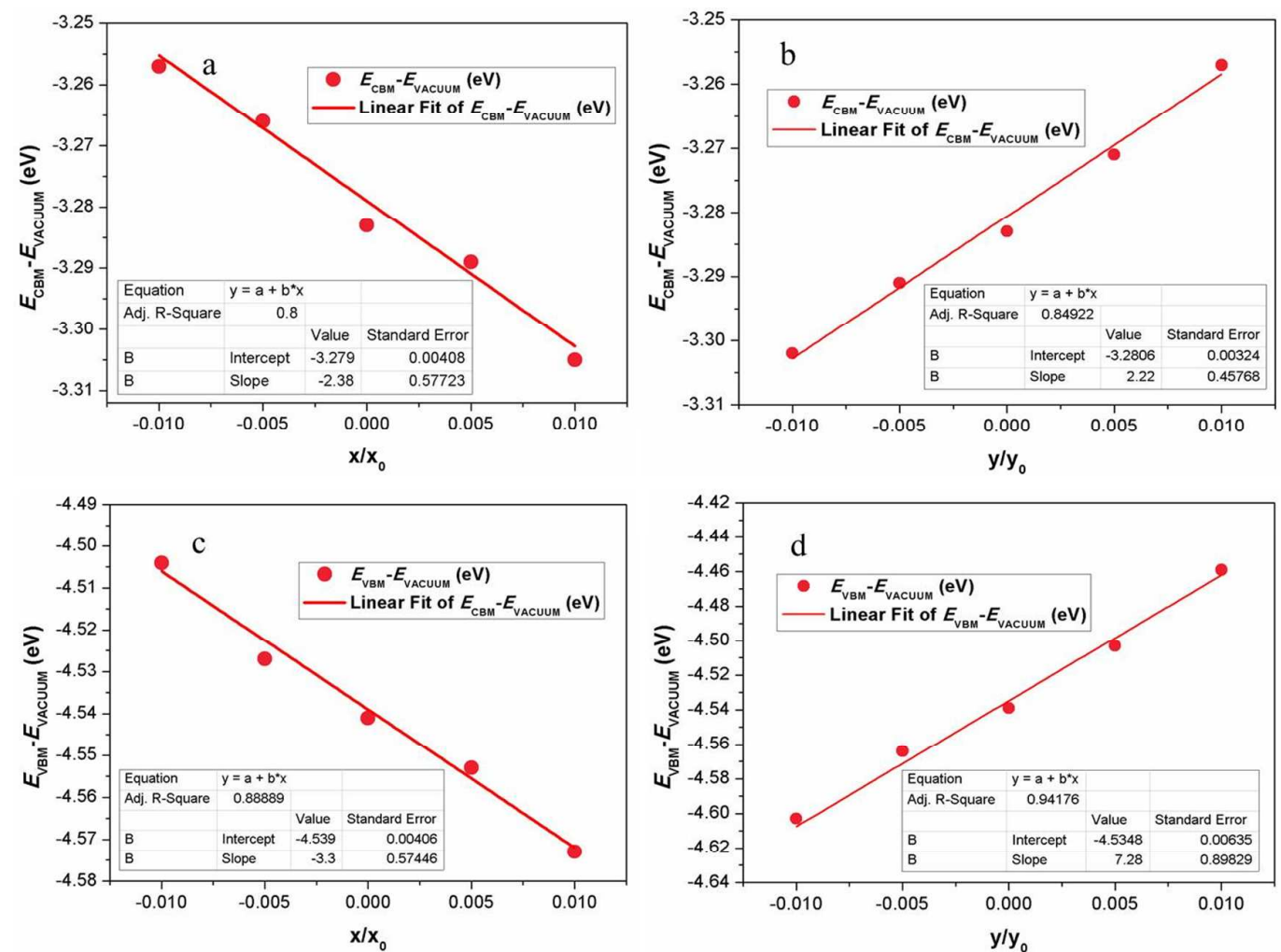

Figure SI6. Band energy of monolayer SnS. (a,b) The CBM of bilayer SnS. (c,d) The VBM of bilayer $\mathrm{SnS}$. 